\title{
Topic Study Group 52: Empirical Methods and Methodologies
}

\author{
David Clarke, Alan Schoenfeld, Bagele Chilisa, Paul Cobb \\ and Christine Knipping
}

TSG 52, "Empirical methods and methodologies," was devoted to explorations of common themes and underlying issues in the use of empirical methods and methodologies. The challenge for the group was defined as follows:

\begin{abstract}
Research in mathematics education employs an extensive range of Methods, Methodologies, and Paradigms (M/M/Ps) in the service of key goals. But which M/M/P combinations help us understand which phenomena, in robust and reliable ways?
\end{abstract}

The group met for four sessions at ICME-13. The first three sessions were devoted to parallel sessions in which the assembled groups explored methodological issues related to the following six goals central to ongoing research in mathematics education:

1. Improving Mathematics Instruction (instructional materials, strategies, organisation, assessment).

2. Understanding the Learning of Mathematics.

3. Understanding the Teaching of Mathematics (teacher beliefs, knowledge, decision-making and professional development).

4. Classroom Processes and Interactions.

5. Mathematics Education and Social Justice.

Co-chairs: David Clarke, Alan Schoenfeld.

Team members: Bagele Chilisa, Paul Cobb, Christine Knipping.

D. Clarke $(\bowtie)$

University of Melbourne, Melbourne, Australia

e-mail: d.clarke@unimelb.edu.au
A. Schoenfeld
University of California, Berkeley, CA, USA
e-mail: alans@berkeley.edu
(C) The Author(s) 2017 
6. Understanding the Role of Culture and Language in Shaping the Teaching and Learning of Mathematics.

These are diverse goals, which might be addressed using research designs that integrate different M/M/P combinations. For each goal, one might be ask: "Suppose you have an hypothesis about this goal. How do you set about evaluating it?" Alternatively, "Suppose you are trying to explain some aspect of individual or group behavior relevant to that goal. How would you characterize and then theorize that behavior?" Or, "How might cultural, historical and political perspectives shape one's understandings of the contingencies related to realizing this particular goal?" One could imagine radically different approaches not only across the six goals, but within each goal. The question, then, was whether the group could, in the final collective discussion, find some degree of coherence in the varied approaches, and produce a larger frame within which the discussions of the six goals could be situated.

That frame emerged as the group considered a broad framing of empirical work in education, as an act of modelling - the idea being that empirical methods and methodologies are, in effect, ways to characterize "real world" phenomena. The challenge, then, was to problematize the enterprise. The challenge was taken up with enthusiasm, which led to some discussions that pushed the boundaries of previous framings.

The group rejected the standard version of the modelling process,

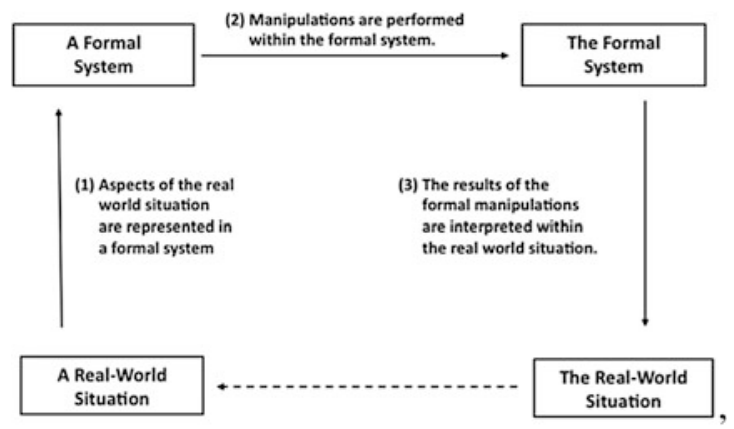

for a more complex one, 


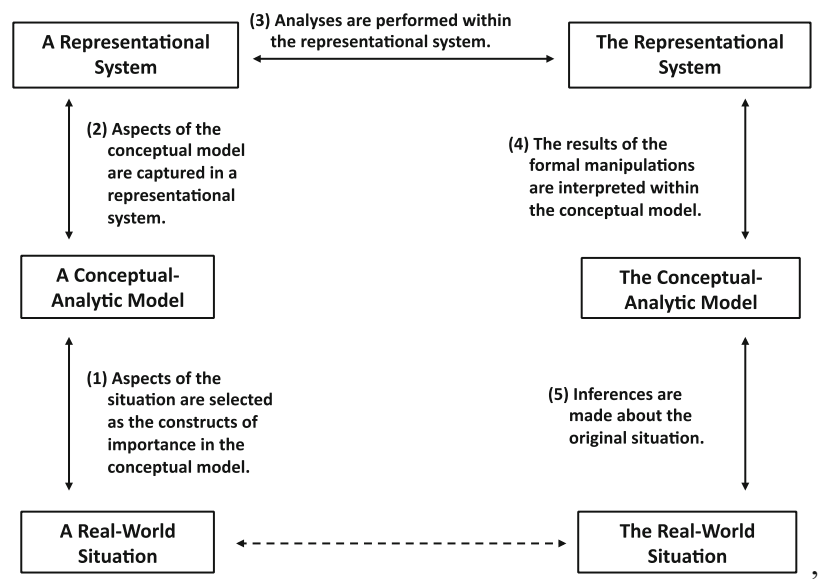

and began to problematize this representation by noting all the ways that empirical work could go wrong - e.g., (arrow 1) for decades of medical research, the "human population" was represented by male experimental subjects, and the results of many studies later turned out not to apply to women. Similarly, (arrow 2) what one decides to consider important in learning makes a big difference-we noted that nations rank differently on TIMSS and PISA, because the two mathematics tests capture different aspects of what might be considered mathematically important. Group members similarly (arrow 3) discussed alternative interpretations of statistical findings - looked at one way, certain results seemed significant, but from another perspective, they were not. Nor were challenges limited to the quantitative realm; questions of how to be confident about interpretations of discourse analyses received attention, as did issues of triangulation and the use of multiple methods. Likewise mapping back from analyses to the "real world" was an issue: the fact that many "significant" laboratory studies did not translate to meaningful learning gains in real classrooms was raised as an issue of concern.

Perhaps the most interesting, and passionate, part of the conversation dealt with what has been called the "political turn" or "socio-political turn" in mathematics education. Members of the group noted that the conceptual analytic models in the second figure often were shaped by tacit social biases - that the "clean" analytic descriptions in the figures could obscure various forms of racism, sexism, gender bias, and more. There was a general concern among the group that the field needs to attend in more explicit ways to the possibility of such bias, and address it in our work. At the same time, there was appreciation for the fact that, although there is a lot more to be done, the field has made tremendous progress since the first ICME in 1969.

Open Access Except where otherwise noted, this chapter is licensed under a Creative Commons Attribution 4.0 International License. To view a copy of this license, visit http://creativecommons. org/licenses/by/4.0/. 